\title{
Comercio justo: La dinámica intermedia entre el productor y el consumidor.
}

DOI: https://doi.org/10.33262/ap.v3i3.1.86

(c) (1) () (2)

Fair Trade: The intermediate dynamics between the producer and the consumer.

Ligia Maricela Niama Rivera. ${ }^{1}$, María Lorena Villacrés Pumagualle. ${ }^{2}$ \& Ruth Genoveva Barba Vera. ${ }^{3}$

\begin{abstract}
.
Fair trade is currently presented as a sustainable alternative for small agricultural and artisan producers in Ecuador, its importance lies in the attitude of international consumers who prefer to buy a product with a fair-trade label to a traditional product; This is based on an attitude of co-responsibility in the face of climate change and poverty in the world. This research analyzes the link to the intermediate dynamics, between the producer and the consumer. Objective: Analyze the sparsely studied space that makes the sales of products possible, establishing the link between producers and generally the citizen who consumes, Methodology: For this, an in-depth bibliographic analysis was carried out to determine the actors in the process and the different factors that affect commercial exchange, which are the mission of organizations, legal forms and types of consumers. Contribution: The visualization of the market dynamics through the types of interactions, conflicts, association dynamics and the role of the State, in the search to "create value with values".
\end{abstract}

Keywords: Fair Trade; Market Dynamics; Producer; Consumer; Mercantile exchange

\footnotetext{
${ }^{1}$ Escuela Superior Politécnica de Chimborazo, Facultad de Informática y Electrónica. Riobamba, Ecuador. lniama@espoch.edu.ec, https://orcid.org/0000-0002-1818-0041

${ }^{2}$ Escuela Superior Politécnica de Chimborazo, Facultad de Informática y Electrónica. Riobamba, Ecuador. marial.villacres@espoch.edu.ec, https://orcid.org/0000-0002-5909-9629

${ }^{3}$ Escuela Superior Politécnica de Chimborazo, Facultad de Informática y Electrónica. Riobamba, Ecuador. rbarba@espoch.edu.ec, https://orcid.org/0000-0003-0272-171X
} 


\section{Resumen.}

El comercio justo se presenta en la actualidad como una alternativa sustentable para los pequeños productores agrícolas y artesanales en el Ecuador, su importancia radica en la actitud de los consumidores internacionales que prefieren comprar un producto con etiqueta de comercio justo a un producto tradicional; esto en función de una actitud de corresponsabilidad frente al cambio climático y a la pobreza en el mundo. En esta investigación se analiza la vinculación a la dinámica intermedia, entre el productor y el consumidor. Objetivo: Analizar el espacio escasamente estudiado que hace posible las ventas de los productos, estableciendo el vínculo entre los productores y generalmente el ciudadano que consume, Metodología: Para ello se realizó un análisis bibliográfico a profundidad para determinar los actores del proceso y los diferentes factores que inciden en el intercambio mercantil, que son la misión de las organizaciones, las formas legales y los tipos de consumidores. Aporte: La visualización, de las dinámicas del mercado a través de los tipos de interacciones, los conflictos, las dinámicas de asociación y el papel del Estado, en la búsqueda de "crear valor con valores"

Palabras claves: Comercio justo, dinámica de mercados, productor, consumidor, intercambio mercantil.

\section{Introducción.}

Entre las tendencias éticas recientes orientadas al consumo sostenible, el Comercio Justo (CJ) es probablemente una de las iniciativas de más rápido crecimiento y una de las más prometedoras. Aunque las ventas de CJ aún no superan el 0,01\% del comercio total del mundo, su tamaño y taza de crecimiento es mucho más que un fenómeno anecdótico. Simultáneamente al éxito que están teniendo los productos de comercio justo, las investigaciones vinculadas a este fenómeno se han incrementado en los últimos años.

En este sentido se ha trabajado mucho en los dos extremos de la cadena del CJ productores y consumidores, los intermediarios de la cadena, principalmente los operadores y motivadores del proceso han recibido menos atención. La supuesta razón es que estas organizaciones vinculan la demanda y la oferta en condiciones precisas determinadas por la etiqueta o el código $\mathrm{CJ}$ con el que hacen alianzas como el caso de la Fundación Maquita, por lo tanto, puede parecer menos importante considerar la forma en que estas organizaciones intermediarias están estructuradas.

Sin embargo, lejos de ser instituciones que realizan actividades de CJ de forma homologada estas organizaciones presentan estructuras, objetivos y formas de funcionamiento muy diversas. Los pioneros del movimiento han sido principalmente organizaciones sin fines de lucro y cooperativas con una fuerte atención a la regulación (el establecimiento de nuevas reglas para llevar a cabo el comercio de manera justa), educación (las iniciativas destinadas a sensibilizar a los consumidores) y al desarrollo 
(una estrecha relación con los productores del campo), estos pioneros del comercio justo a menudo se denominan Organizaciones de Economía Popular y Solidaria (OEPS).

El desarrollo inicial del CJ por parte de las OEPS ha atraído a un número creciente de empresas integradoras "con fines de lucro" en los últimos años, estas empresas han surgido principalmente en el área de distribución (supermercados que venden productos CJ), pero también en la importación y transformación de bienes de comercio justo (por ejemplo, procesadoras de plantas aromáticas o empresas de chocolate). Como paralelo a la integración de CJ, las OEPS, a su vez, han evolucionado hacia un perfil más comercial, como resultado, el CJ ahora aparece como un mercado de forma mixta donde coexisten diferentes tipos de actores, esta convivencia induce a dinámicas de mercado específicas que merecen una atención especial.

Así, los propósitos de este artículo son (1) comprender los factores que han llevado a CJ a convertirse en un mercado de forma mixta y (2) proponer algunos senderos para comprender la dinámica del mercado que resulta de las interacciones entre los diferentes tipos de actores. En la primera parte de este articuló se define brevemente el CJ, sus diferentes dimensiones y su panorama organizacional, en la segunda parte se hace alusión, a los factores teóricos emergentes que describen a los tipos de organización (sin fines de lucro, cooperativa y con fines de lucro) buscando vincular a estos factores en el contexto de la CJ, este análisis permite capturar las especificidades de cada tipo de operador con respecto al CJ y, así, tener una mejor comprensión de la dinámica del mercado en el sector, a través de la competencia, el conflicto y la asociación.

\section{Metodologia.}

La realización de este artículo, se basa en un diseño no experimental, de tipo de campo, de nivel descriptivo como proyecto especial, como material de apoyo educativo, se pretende exponer la dinámica del CJ, tratando de adaptar la comprensión empírica de los teóricos en el área a la realidad económica local, para el efecto se realizó una revisión bibliográfica, a través de los repositorios digitales y bibliotecas virtuales de Scopus, Dialnet, Scielo, Google académico, se buscaron artículos que integran la variable sobre la Dinámica del Comercio Justo.

La búsqueda se realizó en los idiomas español e inglés, los criterios de exclusión se basaron en tres filtros, el primero relacionado en función de las palabras claves de búsqueda SEO, obteniéndose 83 artículos, el segundo filtro relacionado a una lectura rápida del resumen de los trabajos y considerando en lo posible una fecha tope de 5 años, a excepción de algunos trabajos que por sus características ameritaron se considerados, luego de este filtro los documentos se redujeron a 31, el tercer filtro se relacionó a una lectura rápida de los artículos completos, quedando 17 artículos definitivos sobre los cuales se basa el trabajo. Se consideraron para los ejemplos datos obtenidos de la Fundación Maquita, en base a una encuesta realizada por la ESPOCH, sobre la integración del CJ a la Institución. 


\section{Marco Teórico}

\section{El comercio justo y sus actores}

La definición que reúne más consenso y es la más utilizada por actores de campo y académicos es la propuesta por Moore (2004), citado por Cayón, et al., (2016) firma que: El CJ es una especie de coalición comercial, fundamentada en el diálogo, la rendición de cuentas y el respeto mutuo, que busca una mayor equidad en el comercio internacional, contribuye al desarrollo sostenible ofreciendo mejores condiciones comerciales y asegurando los derechos de los productores y trabajadores marginados, especialmente los agricultores y artesanos.

Las organizaciones de CJ (respaldadas por los consumidores) participan activamente en el apoyo a los productores, la sensibilización y la campaña por cambios en las reglas y prácticas del comercio internacional convencional. De esta definición y de la observación del sector se desprende que la definición de CJ es un concepto altamente multidimensional con el que todos están de acuerdo, estas múltiples dimensiones pueden organizarse de diferentes formas (Egas, 2017), de tal manera que los elementos que constituyen esta conceptualización de CJ se pueden agrupar en cuatro categorías principales:

\section{Comercio}

Aunque sea "justo", se está hablando de "comercio", por esta razón los operadores de CJ realizan actividades de mercado, como importaciones o distribución, similares a cualquier iniciativa comercial (Coscione, 2017). El posicionamiento en el mercado dentro de las misiones de los operadores de CJ, sin embargo, puede variar, desde ser un medio para producir más desarrollo entre los productores, hasta constituirse en un objetivo en sí mismo. Al analizar el producto CJ, la dimensión "comercial" se puede equiparar al carácter material del bien, de hecho, no es el producto en sí mismo lo que es "justo": nada distingue físicamente a una fruta $\mathrm{CJ}$ de una convencional, más bien, es el conjunto de características invisibles vinculadas a las condiciones bajo las cuales se ha producido y comercializado el bien (Jaime, et al., 2019)

\section{Justicia}

Por lo tanto, la dimensión "justa" constituye el atributo específico y poderoso, aunque invisible, de los productos CJ, que la diferencia de los productos que no lo son (Egas, 2017). Esta característica "justa" está vinculada tanto a los productores como a los importadores de productos de CJ, mientras que los primeros deben respetar una serie de criterios organizativos (pequeños productores, toma de decisiones democrática, no discriminación, etc.), los segundos tienen derecho a condiciones comerciales específicas (precio justo, prima social, prefinanciación, relación a largo plazo, entre otras).

El precio justo consiste en un " margen de beneficio anticíclico " que puede alcanzar más del $100 \%$ del precio de mercado (por ejemplo, el café, cacao, y otros productos 
ecuatorianos), y que es proporcionado por los importadores de $\mathrm{CJ}$ a las organizaciones de productores de primer nivel $\mathrm{y}$, en última instancia, a los productores individuales, quienes se oponen a esta regla de precios dicen que no se trata de una compensación del mercado, que crea un exceso de oferta y que constituye una distorsión de los mecanismos del mercado.

Sus defensores tienen dos contraargumentos principales: (1) el precio de mercado no es competitivo y refleja la estructura monopsonística / oligopsonística de los intercambios entre productores e intermediarios locales (Camps \& Fregoso, 2019). En estos casos, un precio mínimo más alto (análogo al creado por las OEPS) es la solución a esta falla específica del mercado; (2) el producto CJ (por ejemplo, café CJ) es una variedad nueva y distinta del producto y, como tal, tiene su propio precio de mercado determinado por el equilibrio entre la oferta y la demanda de los consumidores interesados (Coscione, 2017), en este sentido, el comercio justo se puede interpretar verdaderamente como una forma de innovación de productos.

La investigación sobre CJ ha tratado de determinar si el precio justo y las otras herramientas de $\mathrm{CJ}$ conducen efectivamente a un mejor desarrollo socioeconómico para los productores que en el caso del comercio estándar, es decir, si el CJ conduce a una menor desigualdad que el comercio estándar al aumentar las remuneraciones de los productores Stefánski (2016), sostiene que este es el caso solo cuando la demanda de productos es inelástica y los consumidores tienen preferencias de equidad. Ramos y Reverón (2018), sin embargo, sostienen que su enfoque es demasiado estrecho ya que no toma en cuenta características no monetarias de la actividad de CJ (prefinanciamiento de la producción, provisión de bienes públicos locales, promoción de la conciencia del consumidor, etc.).

\section{Educación}

El Comercio Justo también puede verse como una herramienta para la educación de los consumidores. Algunos operadores de CJ, principalmente OEPS, realizan campañas de educación dirigidas a los consumidores, estas campañas están destinadas a promover el CJ y, más globalmente, educar a los ciudadanos (y gobiernos) sobre hábitos de consumo más respetuosos con el medio ambiente y las personas, especialmente con los productores campesinos, incluso si todos los operadores de CJ no realizan actividades educativas, se puede asumir que los consumidores de $\mathrm{CJ}$ financian una parte de estas actividades a través de sus compras.

\section{Regulación}

En los orígenes del CJ, existe la intención de establecer un nuevo marco de regulación para los intercambios comerciales, este marco se aplica, en primer lugar, dentro de las asociaciones de CJ a través de una serie de criterios específicos, además, el marco de regulación del CJ también tiene como objetivo influir en las prácticas comerciales dominantes dentro de los intercambios internacionales. El diseño y el control de las normas específicas dentro del sector de CJ ha sido llevado progresivamente por 
organizaciones vinculadas al CJ específicas que no realizan comercio de productos básicos, sino que se enfocan en la creación y el control de las normas de CJ (esto se conoce como el " etiquetado").

\section{Proceso}

¿Cómo caracterizar estas dimensiones desde un punto de vista económico?

- La característica material del producto CJ es de ser bien privado clásico, al igual que cualquier bien de consumo, el producto en su dimensión física es financiado y consumido por personas en las ciudades y producido por los cultivadores o artesanos que generalmente se encuentran en el campo.

- El carácter "justo" del producto, aunque involucre elementos materiales, es un bien de confianza, de hecho, la aplicación concreta de los principios de CJ por parte de las empresas es algo que el consumidor no puede observar directamente, dado que los beneficiarios de la venta (pequeños productores) se ubican lejos de los que la financian (Los consumidores en los grandes centros poblados), existe una asimetría de información que requiere un cierto nivel de confianza por parte de los consumidores. Vale la pena señalar que también los consumidores (y no solo los productores) son beneficiarios de la dimensión de equidad del CJ, los recientes avances teóricos en la economía experimental han documentado que la equidad y la aversión a la inequidad juegan un papel importante en las preferencias de los consumidores (Roth, 2020), los consumidores con tales preferencias compran productos de CJ no por puro altruismo sino para satisfacer sus preferencias específicas.

La educación de la ciudadanía hacia hábitos de consumo más sostenibles y el establecimiento de nuevas reglas para los intercambios comerciales son dos elementos que no pueden dividirse en unidades de consumo separadas (no exclusividad) y para los que es imposible excluir el consumo mediante el pago de un precio (no rivalidad) (De León, 2019)

Por tanto, parece que CJ se compone de dimensiones privadas, asociativas y de confianza. La Tabla I resume los productores, los beneficiarios y los financiadores de estas diferentes dimensiones, es probable que el carácter multidimensional juegue un papel importante para explicar la diversidad de organizaciones que participan en el CJ (Martínez, et al., 2019).

Tabla 1. Las diferentes dimensiones del Comercio Justo

\begin{tabular}{llll}
\hline & \multicolumn{1}{c}{ Naturaleza } & \multicolumn{1}{c}{ Productores } & \multicolumn{1}{c}{ Beneficiarios } \\
\hline Comercio - bien material & Bien Privado & $\begin{array}{l}\text { Pequeños productores } \\
\text { agropecuarios y } \\
\text { artesanales }\end{array}$ & $\begin{array}{l}\text { Consumidores de las } \\
\text { grandes ciudades }\end{array}$ \\
Equidad - característica justa & $\begin{array}{l}\text { Bien de } \\
\text { Asociación }\end{array}$ & Organizaciones & $\begin{array}{l}\text { Productores } \\
\text { Consumidores }\end{array}$
\end{tabular}


Tabla 1. Las diferentes dimensiones del Comercio Justo (continuación)

\begin{tabular}{llll}
\hline & Naturaleza & Productores & Beneficiarios \\
\hline Educación & Bien Público & OEPS's & Consumidores \\
Regulación & & Etiquetadores OEPS's & Productores
\end{tabular}

De hecho, como se explicó anteriormente, se puede suponer que los consumidores no compran productos de CJ por puro altruismo, sino que obtienen un retorno emocional o psicológico de la compra de CJ en términos de refuerzo de la identidad propia, evitación de la culpa o saciedad de valores.

Fuente: Trabajo de campo

Elaborado por: Los autores

\section{La diversidad dentro del panorama del Comercio Justo}

Los operadores de CJ se pueden clasificar de acuerdo con diferentes criterios. En este contexto, se propone diferenciar estas organizaciones en función de sus (1) misiones, (2) formas legales y (3) tipos de consumidores atendidos, tres criterios que pueden estar parcialmente superpuestos.

\section{Misiones}

Todos los participantes de CJ no necesariamente persiguen conjuntamente las cuatro dimensiones presentadas anteriormente, si bien el "comercio" y la "equidad" son los requisitos mínimos para todos los participantes, se pueden observar diferencias entre las organizaciones que se centran en el comercio y las organizaciones que tienen una visión más amplia del CJ.

Así, se pueden distinguir dos tipos de operadores de CJ:

Organizaciones comerciales alternativas que se dedican total o principalmente al CJ, las OEPS han construido las bases del movimiento CJ y siguen desempeñando un papel fundamental en su desarrollo cualitativo (pero también cuantitativo) (Sama, Crespo, \& Mesías, 2019).

Las empresas que han incorporado la importación, transformación y / o distribución de productos CJ a sus actividades preexistentes, gracias al desarrollo del etiquetado CJ, estas empresas secundarias han adoptado el CJ frente a la presión de los consumidores para encontrar una oportunidad de mercado (Nicolls \& Opal, 2005, p. 80).

Los canales de supermercados y las empresas alimentarias multinacionales son ejemplos típicos de este tipo de empresas, para quienes los productos CJ sólo representan una parte de sus ventas. Nicholls y Opal (2005) identifican varios criterios que diferencian a las OEPS de estas grandes empresas comercializadoras de productos.

Dos de ellos parecen particularmente adecuados para caracterizar a las OEPS: (1) La mayoría de los productos se comercializan bajo los principios del CJ y (2) existe un compromiso organizacional con el CJ como objetivo principal (en lugar de maximizar las ganancias). 


\section{Formas legales}

Históricamente, las OEPS han surgido bajo formas "no capitalistas" o "tercer sector", como organizaciones sin fines de lucro y cooperativas. Sin embargo, en los últimos años, las OEPS también han adoptado formas comerciales "convencionales". Por lo que, a estas organizaciones, se las puede denominar con fines de lucro", aunque las OEPS, no busquen principalmente obtener ganancias. Por lo tanto, las OEPS que se encuentran en la categoría de "con fines de lucro" pueden considerarse empresas comerciales que no buscan la maximización de los beneficios, lo que las acerca a las organizaciones del tercer sector o "empresas sociales" (Cosciene \& Mulder, 2017).

En el Ecuador las OEPS, son amparadas por la Constitución como ley principal y por leyes secundarias que se relacionan con el comercio justo: La Ley Orgánica de Economía Popular y Solidaria (LOEPS), aprobada en abril de 2011, el Código Orgánico de la Producción, Comercio e Inversiones, expedido en noviembre de 2010; y el Código Orgánico de Organización Territorial, Autonomías y Descentralización (COOTAD), aprobado en agosto de 2010.

Sin embargo del reconocimiento jurídico no existe una normatividad que defina adecuadamente que se entiende por $\mathrm{CJ}$, por lo que no se puede establecer de forma precisa que instituciones están a cargo de las funciones normativas de regulación y control, fomento y promoción del C J, por el contrario, se encuentra, dispersión de las instituciones vinculadas, manejo del tema de forma declarativa y con posiciones coyunturales indeterminadas, escasa articulación con una débil coordinación entre instituciones estatales, y lo que es peor un desconocimiento generalizado de la sociedad y el Estado, sobre le CJ la problemática de sus actores.

\section{Tipos de consumidores}

Los análisis empíricos sobre los comportamientos de los consumidores de CJ muestran la existencia de algunas diferencias entre los consumidores que compran productos de $\mathrm{CJ}$ en puntos de venta específicos (Tiendes de venta de productos con etiqueta CJ que a menudo son propiedad de las OEPS) y los consumidores que compran en empresas tradicionales con fines de lucro como los supermercados (Egas, 2017). Primero, los consumidores que se preocupan más por la distancia a las tiendas y que están más preocupados por los horarios de apertura y las ventas en línea tienen más probabilidades de comprar en los supermercados, por lo tanto, es probable que las empresas con fines de lucro que venden en los supermercados o que son ellas mismas distribuidoras atiendan a un tipo de consumidores diferente al de los mercados con etiquetas de $\mathrm{CJ}$, de hecho, los mercados de productos con etiqueta de CJ siguen siendo lugares de venta donde la educación y la conciencia de los consumidores sobre cuestiones sociopolíticas son mucho mayores, por lo que son preferidos por los consumidores "activistas sociales".

En segundo lugar, dado que las principales empresas con fines de lucro tienen economías de escala superiores, pueden vender productos $\mathrm{CJ}$ a precios más bajos, por lo tanto, en la lógica del modelo que se discutirá en la sección "competencia en el sector del CJ", pueden 
atraer consumidores con una compensación diferente entre precio y contenido ético del producto, en este sentido, enfatizando el panorama de alguna manera, se podría decir que los consumidores que requieren que la organización vendedora sea completamente activa en CJ, que aceptan primas más altas y que se preocupan más por las cuestiones de regulación, por lo que es más probable que por la información sobre los productores compren en las tiendas con certificación CJ, mientras que los consumidores que dan más importancia al precio y a la característica que no es del precio, son más propensos a comprar en los supermercados.

Como se mencionó anteriormente, un número creciente de OEPS son de hecho " grupos " que reúnen diferentes entidades en una sola: una organización sin fines de lucro y una cooperativa, en el Ecuador han surgido muchas de estas organizaciones de entre las cuales podríamos nombrar las más representativas como ejemplos: La Corporación del Gruppo Salinas, El Fondo Ecuatoriano Populorum Progeressio (FEPP) o la Fundación Maquita, todas ellas con varios ejes de acción relacionados fundamentalmente con la producción agropecuaria, la trasformación de materias primas para la elaboración de productos alimenticios y artesanales con etiqueta verde, el cooperativismo y el turismo comunitario, entre muchas otras actividades, asociadas al desarrollo comunitario y al CJ.

En cuanto a los principales actores del mercado que se han interesado en el CJ, son principalmente empresas con fines de lucro, desde pequeños negocios hasta corporaciones multinacionales como la Corporación Favorita (Super Maxi y Akí), Corporación el Rosado (Mi Comisariato), El Grupo Narváez (Almacenes Tía, Grupo de empresas ecuatorianas "Santamaría" (Supermercados Santa María), entre las más conocidas. Sin embargo, también es posible que algunas OEPS y las cooperativas actúen como empresas secundarias, a pesar de estas excepciones, para la claridad de este análisis $\mathrm{y}$ con el fin de estudiar las características dominantes de los productores y los consumidores, consideraremos que las OEPS son organizaciones del tercer sector y que estas empresas tienen un beneficio maximizando su objetivo.

\section{Cooperativas}

La forma de cooperativa está particularmente extendida entre las organizaciones de productores de economía popular y solidaria, las razones que explican la agrupación de productores en cooperativas se encuentran en la configuración de los mercados locales, estos mercados se caracterizan por: un número elevado de proveedores para un número reducido de compradores, y una situación de asimetría de información, de hecho, en los mercados locales de productos primarios como quinua, chocho, café o cacao, hay muchos productores individuales para unos pocos compradores situación que se evidencia en la Fundación Maquita que tiene registrados 1461 proveedores, la mayoría de ellos son productores de hortalizas, lácteos y en especial, cereales como la quinua y el chocho (ESPOCH, 2021). Por ese hecho estos compradores tienen fuertes incentivos para aprovechar su poder de mercado con el fin de abusar de los productores, su poder de mercado se ve reforzado por las asimetrías de información sobre los precios y la calidad 
de los bienes: es fácil engañar a un productor individual que no tiene información sobre los precios del mercado y sobre la calidad de su producción.

En este contexto, la creación de una cooperativa es una solución que permite a los pequeños productores controlar los precios a los que venden sus bienes y, así, evitar los altos costos que asumirían si realizaran sus transacciones de manera individual en el mercado (Lusnich, 2020)

La inclusión de la cooperativa en la cadena CJ refuerza la apropiación del poder de mercado por parte de los productores, de hecho, el CJ permite a estos productores acceder a los mercados de exportación y desarrollar capacidades organizativas. Así, desde un punto de vista económico, el CJ puede justificarse por su apoyo a las cooperativas locales como soluciones a las fallas de mercado que enfrentan los pequeños productores (Nicolls \& Opal, 2005)

\section{Dinámica de mercado}

Por un lado, tenemos las OEPS (principalmente organizaciones sin fines de lucro y cooperativas) que generalmente no solo están activas en el comercio, sino también en actividades de educación, negociación y desarrollo, algunos de ellos tienen voluntarios y muchos dependen de subvenciones de ONG, además de sus ingresos comerciales, por otro lado, las empresas principales con fines de lucro) están involucradas en el sector con el comercio como su actividad principal, al ser conscientes de la existencia de diversos tipos de organizaciones con diferentes objetivos activos en el mismo sector, la pregunta es: ¿cómo interactúan estas organizaciones entre sí y cuáles son los resultados de estas interacciones en el mercado CJ?

\section{Tipos de interacciones}

El Comercio Justo puede calificarse como un "mercado de forma mixta" (Ruíz, et al., 2017), es decir, un mercado en el que coexisten proveedores con fines de lucro, sin fines de lucro y, en ocasiones, gubernamentales, la literatura sobre mercados de forma mixta se centra principalmente en la competencia entre proveedores de diferentes tipos, considerándolos como adversarios que compiten por el mismo tipo de productos (Castelo, et al., 2021; Ramos \& Reverón, 2018; Martínez, et al., 2019; Cosciene \& Mulder, 2017)

Sin embargo, la competencia no es el único tipo de relación que puede vincular a organizaciones de diferentes formas. Otros dos tipos de interacciones merecen atención: el conflicto y la asociación (Coscione, 2017). Se describe a continuación en detalle la dinámica de la competencia entre las OEPS (generalmente organizaciones sin fines de lucro o cooperativas) y las empresas secundarias (generalmente con fines de lucro), sobre la base los conceptos teóricos anteriores, se analizan la competencia en bienes de comercio justo considerando su "dimensión justa" como una cualidad socialmente responsable. 
Luego, se observa la competencia entre diferentes tipos de actores que puede desencadenar conflictos y comportamientos de diferenciación, el conflicto entre los comercios con certificación de CJ, los importadores y las organizaciones de etiquetado constituye un ejemplo sorprendente de ello, luego se ilustra hasta qué punto los operadores de CJ de diferentes tipos pueden desarrollar dinámicas de asociación, finalmente, se analiza el papel del gobierno y hasta qué punto sus intervenciones podrían influir en la dinámica de los diferentes actores que conviven en el mercado de CJ.

\section{Conflicto}

El conflicto se observa cuando las organizaciones sin fines de lucro y con fines de lucro se oponen entre sí más allá de la competencia comercial (Martínez, et al., 2019), Estos conflictos a menudo se refieren a importantes cuestiones ideológicas. Por lo general, las organizaciones sin fines de lucro " políticas " (como las ONG) plantean preocupaciones sobre las prácticas comerciales de las multinacionales que se consideran " socialmente irresponsables ", de manera opuesta, las empresas dominantes a veces atacan a las organizaciones sin fines de lucro debido a sus exenciones fiscales, sus subvenciones públicas. (provocando "competencia desleal") o, precisamente, como respuesta a las campañas de denuncia de las ONG.

Uno de los conflictos observados en el desarrollo del mercado CJ es el que se da entre las los mercados con etiqueta CJ importadores y organizaciones de etiquetado, la simple inspección de las diferentes posiciones de estos actores en la cadena de valor ayuda a comprender que sus objetivos estratégicos heterogéneos abren el camino al conflicto, las tiendas con certificado son minoristas dedicados a la venta de productos $\mathrm{CJ}$ que pueden ayudar a las asociaciones importadoras a reducir los cuellos de botella en la distribución que crean una brecha entre la demanda potencial y la real, por el contrario, dichos canales sufren la competencia de cualquier otro canal de distribución diferente (supermercados, promotores individuales de CJ, etc.).

Finalmente, las asociaciones de etiquetado pueden verse tentadas a certificar nuevos productos con características CJ creadas por imitadores que maximizan las ganancias, el hecho que desencadena el conflicto es la imitación parcial de las multinacionales alimentarias y la decisión de las grandes cadenas de distribución de vender productos de $\mathrm{CJ}$, esta decisión representa un negocio potencial adicional para organizaciones de etiquetado a la vez que constituye una amenaza competitiva para los importadores pioneros y las tiendas mundiales (Cosciene \& Mulder, 2017).

\section{Dinámicas de asociación}

Las asociaciones entre empresas sin fines de lucro y con fines de lucro han recibido una atención creciente en la literatura (Cayón, et al., 2016; Cosciene \& Mulder, 2017; Jaime, et al., 2019; De León, 2019; Ruíz, et al., 2017), la asociación puede definirse como la colaboración entre empresas independientes en un espacio y tiempo económicos determinados para el logro de metas mutuamente definidas (Coscione, 2017). 
A lo largo de la cadena de valor de CJ, se han desarrollado varias formas de asociación entre diferentes actores. Como es fácil de imaginar, es más probable que las asociaciones sean desarrolladas por participantes que están integrados verticalmente, uno de los modelos de colaboración más comunes ocurre entre OEPS, minoristas con fines de lucro y tiendas certificadas CJ, las OEPS suelen ofrecer a los nuevos supermercados de CJ esquemas de franquicia en los que la creación de un nuevo establecimiento se apoya desde un punto de vista financiero y organizativo con la condición de vender no menos de una parte determinada de los productos importados por el OEPS "franquiciador".

Además, cuando las OEPS celebran acuerdos de venta con minoristas con fines de lucro, generalmente solicitan a estos últimos que incluyan tiendas certificadas en la empresa (bajo la forma de participación en las ganancias o intermediación de productos con minoristas con fines de lucro), vale la pena señalar que, bajo este esquema, es la relación vertical entre las OEPS y el supermercado la que hace cumplir el acuerdo de cooperación entre los dos tipos de minoristas y no su cooperación directa (Bennett, 450)

Las asociaciones entre actores que están en el mismo nivel de la cadena de valor son menos frecuentes, pero ocurren cuando diferentes actores tienen objetivos comunes. Estos objetivos comunes pueden ser promover el CJ y sensibilizar al público en general, en tal caso, la colaboración surge bajo una figura formal o consorcios informales ad hoc, por ejemplo, para organizar campañas publicitarias y educativas o para desarrollar proyectos comerciales comunes (Jaime, et al., 2019).

El segundo tipo de asociación puede ocurrir entre empresas que enfrentan desafíos comunes con una línea de productos específica, dado que estas empresas comunes incluyen elementos de bien colectivos, benefician a todos los participantes y, por lo tanto, son un terreno fértil para la colaboración una vez que se resuelven los problemas de "aprovechamiento libre" en las normas contractuales de los consorcios mencionados anteriormente (De León, 2019).

\section{El papel del Estado}

El Comercio Justo ha crecido sin ninguna ayuda gubernamental específica y, al menos al principio, sin el apoyo de las instituciones públicas, la razón es que, al principio, el CJ era solo una preocupación para un pequeño número de ciudadanos que estaban insatisfechos con los niveles de ayuda al desarrollo y / o de regulación en el comercio internacional, por lo tanto el gobierno no tenía ningún incentivo para intervenir en un asunto que no le interesaba al "votante medio", es en este contexto han surgido fundaciones sin fines de lucro como respuesta de ciudadanos insatisfechos, sin embargo, como el CJ se ha desarrollado enormemente y se ha convertido en una preocupación para el votante medio, el gobierno ahora está mucho más ansioso por interesarse en este sector (Clark , 2017)

Entre las opciones de política disponibles, es poco probable que exista una preferencia fiscal por las OEPS debido a la naturaleza misma de las organizaciones de CJ como empresas sociales que crean bienes de consumo, pero compiten en el mercado con empresas con fines de lucro. Incluso considerando su papel social, es probable que dicha 
política fiscal se considere discriminatoria e injusta en el sentido de que establecería un apoyo ilícito a un participante del mercado específico en la carrera competitiva.

En general y más allá de esta medida concreta, si bien existe cierto consenso en que las autoridades públicas no deben participar directamente en la competencia del CJ, no se puede negar que sus decisiones sobre las reglas del juego pueden tener una influencia crucial en la promoción de la responsabilidad social como característica del mercado competitivo.

Tres son las principales sugerencias de política que se derivan fácilmente de esta consideración, en primer lugar, las reglas de adquisición orientadas al precio pueden ser cruciales, ya que el precio mínimo como criterio competitivo único contrasta definitivamente con el objetivo de promover la responsabilidad social empresarial.

En segundo lugar, la información obligatoria sobre la calificación social de los vendedores y sobre las características sociales de la cadena de valor en los principales puntos de venta sería una regla de costo cero que podría impulsar la competencia en responsabilidad social.

En tercer lugar, en un contexto de competencia en el que algunos participantes proponen diferentes tipos de etiquetas éticas e incluso diferentes versiones de CJ (etiquetas "ligeras"), el propio gobierno puede establecer normas de CJ con el fin de determinar un contenido mínimo para lo que se puede llamar Comercio Justo.

\section{Conclusiones.}

- Este artículo ha investigado la complejidad de la dinámica organizacional dentro del sector de CJ., desde las características inusuales de los productos CJ y cómo estas características explican el surgimiento de una variedad de participantes comerciales.

- Se ha podido comprender que el producto CJ incluye un conjunto de características físicas e inmateriales que lo distinguen de los productos estándar, por lo tanto, el CJ constituye una innovación en el sentido de que permite combinar una dimensión comercial con la equidad y los objetivos sociopolíticos, de tal manera, que la distinción entre estas diferentes dimensiones es crucial para explicar el surgimiento de los actores del CJ bajo diferentes formas legales.

- Se ha documentado la dinámica entre los diferentes tipos de actores a través de tres dimensiones fundamentales: competencia, conflicto y asociación, el primero ha estado representado por la competencia entre las OEPS pioneras y los imitadores parciales con fines de lucro. El segundo ha sido ilustrado por los conflictos de intereses entre los comercios con certificación CJ, importadores y organizaciones de etiquetado.

- Finalmente, incluyendo el rol del Estado con respecto a estas interacciones, se ha analizado en qué medida las interacciones entre los diferentes tipos de actores 
serán cruciales para la supervivencia y fortalecimiento del núcleo del CJ: el de " crear valor con valores. "

- El CJ representa uno de los ejemplos más interesantes de una gran innovación en el funcionamiento de los sistemas económicos contemporáneos. Con su "voto de cartera", los consumidores de CJ también son un desafío a la definición reduccionista estándar de las preferencias del homo oeconomicus. Su disposición a pagar en exceso por las características sociales de productos viene en apoyo de un nuevo conjunto de teorías que ven la justicia, la aversión a la inequidad, la simpatía y el compromiso como importantes motivaciones adicionales de la economía con opciones.

- La característica novedosa del consumo de CJ es que, por primera vez, la equidad y el rechazo a la inequidad no se relacionan con vecinos o compañeros de trabajo sino con productores distantes, documentando así una ampliación significativa del grupo de referencia que los individuos consideran en su elección.

- El Comercio Justo está, en cierto sentido, contribuyendo a mejorar la reputación del mercado, ya que lo convierte no solo en el lugar en el que los intercambios reflejan puramente el poder de negociación desequilibrado y las dotaciones de compradores y vendedores, sino también en el espacio en que es posible contribuir a reducir tales desequilibrios mediante el "intercambio de valores".

\section{Referencias bibliográficas.}

Bennett, E. (450). The Global Fair Trade Movement: For Whom, By Whom, How, and What Next. Lewis \& Clark College, 459-477. Obtenido de https://papers.ssrn.com/sol3/papers.cfm?abstract_id=3872892

Camps, S., \& Fregoso, H. (2019). Microeconomía Ecológica. En A. Almeida , \& J. Matus , Tendiendo Puentes para una sostenibilidad integral (págs. 61-82). México: Universidad Autónoma Metropolitana.

Castelo, M., Guadalupe, S., \& Auquilla, P. (2021). El comercio justo en el contexto del desarrollo sostenible. Visionario Digital, 5(1), 36-51. Obtenido de https://www.cienciadigital.org/revistacienciadigital2/index.php/VisionarioDigita 1/article/view/1535

Cayón , E., García, M., \& Pérez, A. (2016). El perfil del consumidor de comercio justo. Revista de Dirección y Administración de Empresas(23), 75-95. Obtenido de https://addi.ehu.es/handle/10810/20330

Clark , P. (2017). Polítiticas publicas y Comercio Justo en el Ecuador: El Caso de la Estrategia Ecuatorian para el Comercio Justo. Obtenido de https://www. cepal. org/sites/default/files/events/files/patrick_clark__politicas_publicas_y_comercio_justo_en_el_ecuador_0. pdf. 
Cosciene, M., \& Mulder, N. (2017). El aporte del comercio justo al desarrollo sostenible. Santiago de Chile: CEPAL. Obtenido de https://repositorio.cepal.org/handle/11362/42404

Coscione, M. (2017). Comercio Justo y Objetivos de Desarrollo Sostenible. CLAC, 8(19), s/p. Obtenido de https://www.altramerica.info/wpcontent/uploads/2018/03/cjods-1.pdf

De León, A. (2019). Certificación en comercio justo y el bienestar social de los pequeños productores. Oaxaca: Instituto Politécnico Nacional. Obtenido de http://literatura.ciidiroaxaca.ipn.mx/jspui/handle/LITER_CIIDIROAX/412

Egas, F. (2017). Comercio justo o economía solidaria. Revista afese,, 49(29), 1-4.

ESPOCH. (2021). Informe Convenio Fundación Maquita-ESPOCH. Riobamba: ESPOCH.

Jaime, P., Ramírez, L., Ponce, I., \& Huanca, A. (2019). El comercio justo como alternativa para desarrollar estrategias en beneficio de los productores. Dominio de las Ciencias, $\quad 5(3), \quad 589-602$. Obtenido de https://dialnet.unirioja.es/servlet/articulo?codigo $=7154283$

Lusnich, C. (2020). Estrategias de producción sustentable, comercio directo y precio justo en la Economía Social y Popular. Buenos Aires: Observatorio Social sobre Empresas Recuperadas y Autogestionadas,. Obtenido de https://publicaciones.sociales.uba.ar/index.php/osera/article/view/5244

Martínez , L., Vanegas, D., Colmenares, H., \& Rojas, D. (2019). Comercio justo y las oportunidades que representa el modelo para el desarrollo cooperativo. Negonotas Docentes, $\quad 45-53 . \quad$ Obtenido de https://revistas.cun.edu.co/index.php/negonotas/article/view/574

Moore, G. (2004). The Fair Trade movement: Parameters, issues and future research. Journal of Business Ethics(53), 73-86.

Nicolls , A., \& Opal, C. (2005). Fair trade: Market-driven ethical consumption. New York: Sage. Obtenido de https://books.google.es/books?hl=es\&lr=\&id=Hmj6UlKhVDcC\&oi=fnd\&pg=P $\mathrm{P} 10 \& d q=$ Nicholls $+\mathrm{y}+\mathrm{Opal},+2005 \&$ ots=5_7NJ7_p71\&sig=A6pqePdETirTlWta DnTaJuMS150

Ramos, A., \& Reverón, M. (2018). Comercio Justo: un estudio empírico para explorar sus determinantes. Tenerife: Universidad de la Laguna. Obtenido de https://riull.ull.es/xmlui/bitstream/handle/915/12003/Comercio+Justo+un+estudi o+empirico+para+explorar+sus+determinantes+.pdf?sequence $=1$ 
Roth, A. (2020). 1. Introduction to experimental economics. In The handbook of experimental economics. Princenton: Princenton Uninersity Press. Obtenido de https://www.degruyter.com/document/doi/10.1515/9780691213255-003/html

Ruíz, F., Valenzuela, P., \& Rendon, H. (2017). Educación y economía. El comercio justo, alternativa para reducir los índices de pobreza, Ecuador (original). Olimpia: Publicación científica de la facultad de cultura física de la Universidad de Granma, 65-80.

Sama, C., Crespo, E., \& Mesías, F. (2019). ). Análisis de las preferencias de los consumidores españoles hacia la miel de producción social y ambientalmente responsable frente a la de Comercio Justo. Archivos de zootecnia,, 68(264), 495503.

Obtenido

de https://www.uco.es/servicios/ucopress/az/index.php/az/article/view/4988

Stefánski, R., \& Stefánska, M. (2016). Macroeconomic Consequences of the Fair Trade Development for Exporters of Agricultural Commodities, in. En J. Andreani, \& $\mathrm{U}$. Collesi, roceedings of Conference XV International Marketing Trends Conference 2016 Venice,. Paris: Marketing Trends Asociation . Obtenido de www.marketing-trends-congress.com/papers]

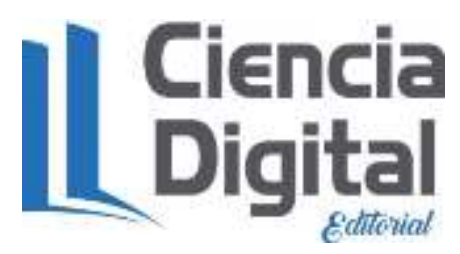




\section{PARA CITAR EL ARTÍCULO INDEXADO.}

Niama Rivera, L. M., Villacrés Pumagualle, M. L., \& Barba Vera, R. G. (2021). Comercio justo: La dinámica intermedia entre el productor y el consumidor. AlfaPublicaciones, 3(3.1), 185-201. https://doi.org/10.33262/ap.v3i3.1.86

\section{Ligital}

El artículo que se publica es de exclusiva responsabilidad de los autores y no necesariamente reflejan el pensamiento de la Revista Alfa Publicaciones.

El artículo queda en propiedad de la revista y, por tanto, su publicación parcial y/o total en otro medio tiene que ser autorizado por el director de la Revista Alfa Publicaciones.
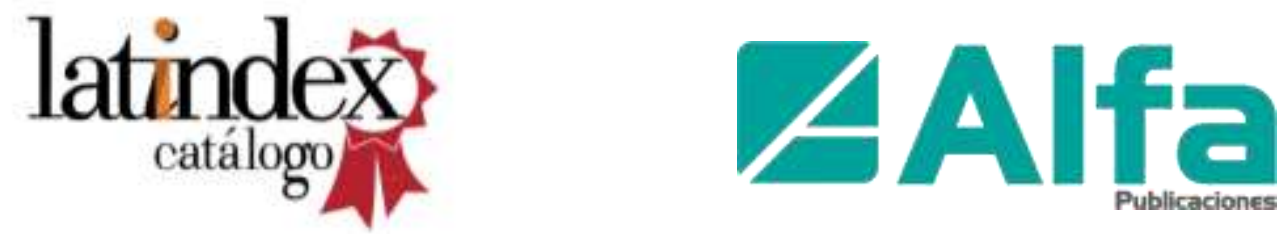